Urología N eurológica y Urodinámica

Arch. Esp. Urol., 58, 4 (316-323), 2005

\title{
FACTORES DE DESCOMPENSACIÓN MICCIONAL Y CISTOCELE.
}

\author{
Jesús Salinas Casado, José María Adot Zurbanoํㅜ, Miriam Dambros, M iguel Vírseda \\ Chamorro ${ }^{2}$, Juan Carlos Ramírez Fernández, Jesús Moreno Sierra, José Marcos Díaz y Ángel \\ Silmi Moyano.
}

Servicios de Urología. Hospital C línico San Carlos. Universidad Complutense. Madrid.

Servicios de Urología. Hospital G eneral Yagüe ${ }^{1}$. Burgos.

Servicios de Urología. Hospital C entral de la Defensa ${ }^{2}$. Madrid. España.

\begin{abstract}
Resumen.- 0 BJETIVO S: Valorar las ca racterísticas clínicas y urodinámicas de una serie de mujeres con residuo postmiccional (micción descompensada) y diferentes grados de cistocele asociado.

MÉTODOS: Se estudiaron 119 pacientes de sexo femenino mediante evaluación clínica, urodinámica, y radiológica (cisto uretrografías). Todas las pacientes fueron sometidas a historia clínica, exploración física uroginecológica (valorando el cistocele, al vasalva, de grado 0 a grado 3), exploración física neurourológica y estudio urodinámico completo. A 50 pacientes (42\%), se les realizó estudio radiológico del tracto urinario inferior (TUI). Se consideró micción descompensada (MD), la existencia de un residuo postmiccional (RP) mayor del $20 \%$ del volumen miccional. La resistencia uretral se midió mediante el URA. La tipificación de la obstrucción estructural se realizó mediante el PURR (clasificación CHESS). El estudio de la obstrucción funcional se realizó mediante el DURR y la EMG perineal (asociada a la flujometría libre). La potencia contráctil del detrusor se valoró mediante el W max, W 80-20 y
\end{abstract}

\footnotetext{
Jesús Salinas C asado. Servicio de Urología Hospital Clínico San Carlos. Prof Martin Lagos S/ N. 28040 Madrid. (España)

duración de la contracción del detrusor. La terminología urodinámica y medidas siguieron las normas de la International Continence Society, ICS. La probabilidad de los diferentes análisis estadísticos se consideró significativa por debajo de 0.05 , a nalizándose las variables cuantitativas mediante el test de la $T$ de Student, y las variables no paramétricas mediante la chi cuadrado de Pearson.

RESULTADO S: La edad media de las pacientes (119), fue de 55.84 años (rango 15-87). En relación con el $R P$ (1 14 flujometrías valorables), fueron etiquetadas de MD, 25 pacientes (21.9\%) y compensada (MC), 89 (74.8\%). Respecto a los datos clínicos, solo existieron diferencias significativas (DS) entre a mbos grupos en la dificultad miccional. En los datos flujométricos, existieron tan solo DS en el percentil (nomogramas de Haylen), del Q $\max (35$ MC vs 22, M D; $p=0,02$ ). En la cistomanometría, se demostró hiperactividad vesical en la MD en 16 casos (64\%) frente a 31 casos (34,8\%) en la MC $(p=0,008)$, que presentó relación con incremento de la resistencia uretral (URA) $p=0,01$. En el estudio $P / F$, se demostró DS en el valor del URA (14.7 MC vs $25.3 M D ; p=0,001)$. Existieron $D S$ en los grados de obstrucción constrictiva (0.5 MC vs $1.1 M D ; p=0,009)$ y compresiva $(0.02 M C$ vs $0.4 M D ; p=0,04$ )(clasificación CHESS). Estos datos no presentaron relación significativa con el grado de cistocele. No existieron DS en los a nálisis de contractilidad isométrica (W max), pero sí en la contractilidad isotónica (W 80-20) y duración de la contracción del detrusor. Estas últimas diferencias si presentaron relación significativa con el grado de cistocele. Los datos de DURR y EMG perine- 
al no arrojaron DS entre ambos grupos. Las alteraciones de la morfología uretral radiológica fueron estadísticamente diferentes entre ambos grupos estando presente en el $10 \%$ de la MC vs $50 \%$ de la MD $(p=0,01)$, aunque no presentó relación estadística $(p=0,64)$, con la obstrucción. La presencia de cistocele, así mismo, no modificó esta dística mente ning uno de todos los da tos anteriores.

CON C LUSIO N ES: La micción descompensada, acompañó tanto a la obstrucción orgánica (más significativamente constrictiva) del tracto urinario inferior, como a la a fectación contráctil del detrusor, pero, mientras que en la primera condición no se relacionó significativamente con la presencia y grado de cistocele, en la segunda si se demostró dicha asociación.

Palabras clave: Cistocele. Residuo Postmiccional. Urodinámica.

Summary.- OBJECTIVES: To evaluate the clinical and urodynamic features of a series of women with post void residual urine (disbalanced voiding ) and various degrees of associa ted cystocele.

METHODS: 119 female patients were studied by clinical evaluation, urodynamics, and imaging tests (VC UG ). All patients underwent history and genitourological examination (evaluating cystoceles from grade 0 to 3), neuro-urological examination, and complete urodynamic study. Fifty patients (42\%) underwent radiological studies of the upper urinary tract Disbalanced voiding was defined as existence of post void residual greater than $20 \%$ of the voided volume. Urethral resistance was measured by URA. Structural obstruction was characterized by PURR (CHESS classification). Functional obstruction was studied by DURR and perineal EM G (associated with flow metry). Detrusor contractile power was evaluated by $W$ max, W 80-20, and duration of contraction. Urodynamic terminology and measurements complied with the International Continence Society (ICS) standards. Statistical significance was established at 0.05. Statistical a nalysis was done by Student's $t$ for quantitative variables, and Pearson's chi-square for non parametric variables.

RESULTS: 119 patients were enrolled. M ean age was 55.84 yr. (range 15-87). Regarding post void residual (114 valid uroflow metry studies), 25 patients were classified as voiding disbalance (21.9\%) and 89 as balanced (74.8\%). Regarding clinical data, there were only significant differences between groups in voiding difficulty. For uroflowmetry, only the percentile of the Maximal flow (Q max) showed significant differences
(35 vs. 22 for balanced/ disbalanced voiding respectively, $p=0.02$ ). Pressure/ volume studies demonstrated bladder hyperactivity in 16 cases $(64 \%)$ in the group of disbalanced voiding and 31 cases (34.8\%) in the normal voiding group $(p=0.008)$, which presented associated with increased urethral resistance $(U R A)(p=$ $0.01)$. In the pressure/ flow study, there were significant differences in the URA (14.7 vs. 25.3, $p=$ $0,001)$. There were statistically significant differences in the degree of constrictive (0.5 vs. 1.1, $p=0.009)$ and compressive (0.5 vs. $1.1 ; p=0.04)$ obstruction (Chess classification). There were not significant differences in the analysis of isometric contractility (W max), but there were in the isotonic contractility (W 80-20) and detrusor contraction duration. These latter differences presented significant association with the degree of cystocele. DURR and perineal EM G data did not show differences between groups. Radiological abnormalities of urethral morphology were statistically different between groups, presenting in $10 \%$ of the patients with normal voiding and $50 \%$ of the disbalanced voiding group, although there was not statistical association with obstruction $(p=0.64)$. The existence of cystocele did not show a statistical association with these variables either.

CON CLUSIO N S: Disbalanced voiding appeared with organic obstruction of the lower urinary tract (constrictive most significantly), as well as detrusor abnormal contractility, but whereas the first was not significantly associated with presence and grade of cystocele, the second showed such association.

Keywords: Cystocele. Post void residual.

Urodynamics.

\section{INTRODUCCIÓN}

La presencia de residuo postmiccional (RP), que define la micción descompensada, no es un dato frecuente en la mujer. Su demostración puede estar en relación, tanto con la existencia de obstrucción del tracto urinario inferior (TUI), como en la afectación contráctil del detrusor. La realización de dichos diagnósticos urodinámicos no están totalmente definidos en la mujer, a diferencia del sexo masculino. Por otra parte, el cistocele, patología muy frecuente en el sexo femenino, ha sido estudiado más en relación con la producción de incontinencia urinaria (por alteración en la fase de llenado vesical), que con el residuo pos- 
tmiccional (alteración de la fase de vaciamiento). De ahí, nuestro interés en estudiar la interferencia del cistocele aislado, en la dinámica miccional, en ausencia de toda patología neurourológica.

\section{MATERIALES Y MÉTODOS}

Se estudiaron 119 pacientes de sexo femenino. Se les realizó estudio clínico, urodinámico, y radiológico (cistouretrografías). Todas las pacientes fueron sometidas a historia clínica, exploración física neurourológica y uroginecológica y estudio urodinámico completo.

La edad media de las pacientes fue de 55,8 años (rango 15-87).

A 50 pacientes (42\%), se les realizó estudio radiológico (cistouretrografía), del tracto urinario inferior (TUI).

El flujo miccional, además de valorar los parámetros de flujo máximo y medio, se interpretó mediante el Nomograma de Haylen para la mujer adulta, que clasifica los valores en percentiles. Se consideró micción descompensada (MD), la existencia de un residuo postmiccional (RP) mayor del $20 \%$ del volumen miccional.

El grado de cistocele (clínico), se consideró 0 o no cistocele, ante la ausencia de prolapso vesical durante la tos. Grado 1, ante descenso leve de la misma; grado 2 cuando se produce descenso, con la tos 0 el valsalva, hasta el introito vaginal y grado 3 , cuando desciende por debajo del introito vaginal. Se excluyeron los casos con asociación de otros prolapsos significativos, así como toda condición patológica neurourológica.

La resistencia uretral se midió mediante el URA (Resistencia Uretral Media). ( $\mathrm{cm} \mathrm{H}_{2} \mathrm{O}$ )

La tipificación de la obstrucción estructural se realizó mediante el PURR (Relación de Resistencia Uretral Pasiva) (clasificación CHESS), en compresiva y constrictiva (Grado 1,2 y 3 ).

El estudio de la obstrucción funcional se realizó mediante el DURR (Relación Uretral de Resistencia Dinámica) y la EMG (Electromiografía) perineal (asociada a la flujometría libre).

La potencia contráctil del detrusor se valoró mediante el W max (Parámetro de contractilidad iso- métrica), W 80-20 (Parámetro de contractilidad isotónica) y duración de la contracción del detrusor (medida por el residuo postmiccional de la capacidad total vesical en el test presión del detrusor / flujo miccional). La terminología urodinámica y medidas siguieron las normas de la International Continence Society, ICS.

Se recogieron los datos en el programa excel 2000 y el tratamiento estadístico se realizó con el paquete SPSS.

La probabilidad de los diferentes análisis estadísticos se consideró significativa por debajo de 0.05 , analizándose las variables cuantitativas mediante el test de la $T$ de Student, y las variables no paramétricas mediante la chi cuadrado de Pearson.

\section{RESULTADOS}

\section{1.- Datos Generales}

Se estudiaron 119 pacientes de sexo femenino, cuya edad media fue de 55,8 años (rango 15-87 años).

El residuo postmiccional (114 casos), tuvo un valor medio de 52,3 $\mathrm{ml}$ (rango 0-600 ml).

Se observó micción descompensada (residuo postmiccional mayor de $20 \%$ del volumen orinado), en 25 casos $(21,9 \%)$, siendo compensada en los 89 casos restantes $(78,1 \%)$. (Figura 1$)$.

Las pacientes con micción compensada $(89$ casos) fueron estudiadas a una edad media de 56,2 años y aquellas con micción descompensada (25 casos), a una edad promedio de 52,6 años. No existieron diferencias entre ambos grupos $(p=0,33)$.

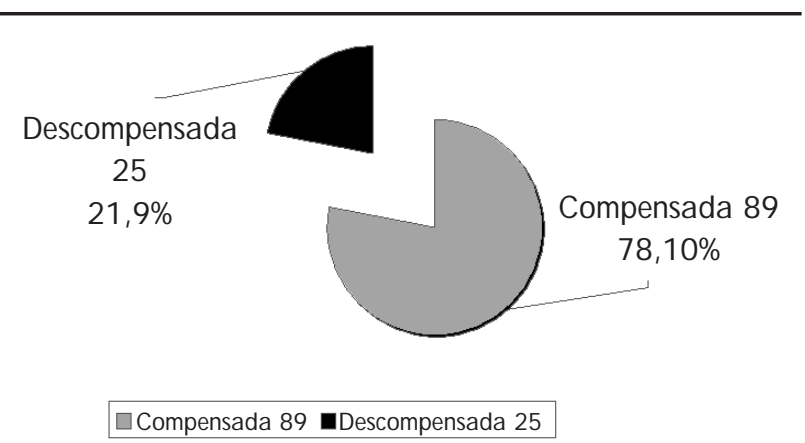

FIG URA 1. Compensación miccional $N=114$ 
El grado de cistocele valorado clínicamente (119 casos), fue 0 (no cistocele), en 37 casos $(31,1 \%$ ), 1 en 30 casos $(25,2 \%)$, 2 en 31 casos $(26,1 \%)$ y 3 en 21 casos $(17,6 \%)$. (Figura 2$)$.

\section{2-. Síntomas del Tracto Urinario Inferior}

De las pacientes interrogadas (113 casos), refirieron dificultad miccional 8 de las 81 pacientes con micción compensada $(9,9 \%)$ y 10 de las 32 con micción descompensada (31,3\%), siendo la diferencia en la proporción, muy significativa $(p=0,0003)$. (Figura 3).

No existieron diferencias significativas entre ambos grupos, en los síntomas funcionales de vaciamiento del Tracto Urinario Inferior (LUTS), como el goteo postmiccional $(p=0,59)$, alteraciones en el caudal miccional (disminuido o entrecortado; $p=0,78$ ), sensación de micción incompleta $(p=0,62)$, así como en infecciones del TUI $(p=0,32)$, y sensación de bulto en la vagina $(p=0,34)$.

\section{3.- Exploración Física Neurourológica.}

$\mathrm{N}$ o se observaron diferencias significativas en la proporción de pacientes con micción compensada 0 descompensada y sensibilidad perineal alterada 0 normal $(p=0,79)$, tono anal $(p=0,79)$, reflejo bulbocavernoso $(p=0,79)$, ni control voluntario esfinteriano $(p=0,79)$.

\section{4.- Datos flujométricos}

$\mathrm{N}$ o se observaron diferencias significativas en el volumen miccional promedio entre ambos grupos $(p=0,4)$.

El residuo postmiccional medio de las pacientes con descompensación miccional (25 casos), fue de

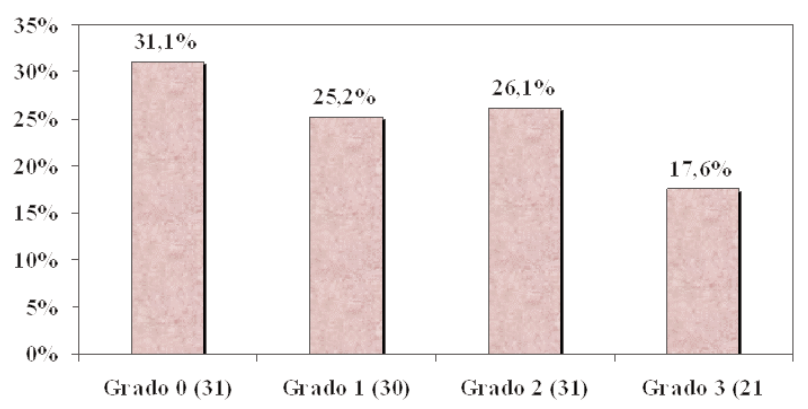

FIG URA 2. G rado clínico de cistocele. $N=119$
$211,6 \mathrm{ml}$ y en las pacientes con compensación miccional (89 casos), fue de $9,4 \mathrm{ml}$.

Respecto a la compensación o descompensación miccional, se observó descompensación miccional en el 31,4\% (11 de 35) de las pacientes sin cistocele (grado 0), en el $13,3 \%$ de las pacientes con cistocele 1 (4 de 30 ), en el 13,8\% de las pacientes con cistocele 2 ( 4 de 29 ) y en el $30 \%$ de las pacientes con cistocele 3 (6 de 20). No existieron diferencias significativas en la proporción de descompensación miccional entre los diferentes grados de cistocele $(p=0,17)$. (Figura 4).

No se observaron diferencias en la actividad EM G perineal durante la flujometría libre $(p=0,23)$, así como con el grado de cistocele, ni en el valor promedio del flujo máximo $(p=0,13)$ ni del flujo medio $(p=0,17)$. Únicamente se demostraron diferencias significativas $(p=0,02)$ en el percentil promedio del $Q \max$ (35 MC vs 22 MD).

\section{5.- Datos cistomanométricos.}

Se observaron diferencias significativas en la proporción de hiperactividad en las pacientes con MD frente a las pacientes con MC $(p=0,008 ; 16$ de 25 casos de MD (64\%), frente a 31 de 89 en pacientes con MC $(34,8 \%)$. Dicha hiperactividad presentó significación estadística $(p=0,01)$ con el incremento de la resistencia uretral, pero no con el grado de cistocele.

\section{6.- Estudio presión flujo \\ A) Tipo de micción.}

Respecto al tipo de micción, no se observaron diferencias entre las pacientes con compensación o descompensación miccional, grado de cistocele y tipo de mic-

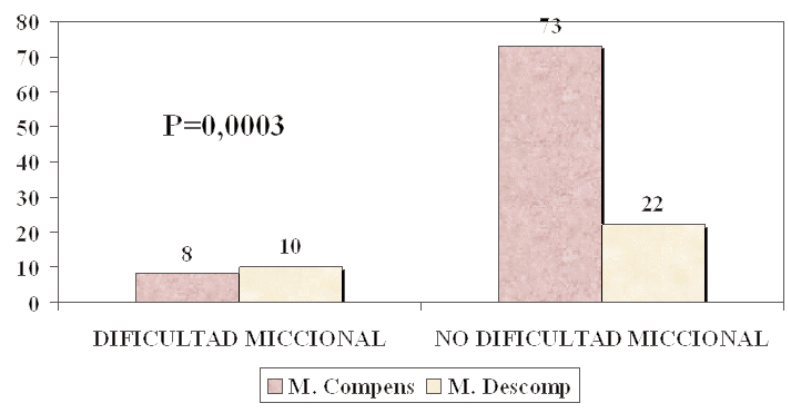

FIG URA 3. Dificultad miccional / Compensación miccional. $N=113$ 
ción (voluntaria, involuntaria o prensa abdominal) $(p=0,06)$, ni ayuda de prensa abdominal para realizar la micción $(p=0,64)$.

\section{B) Parámetros de resistencia uretral.}

El parámetro URA (resistencia uretral orgánica), tuvo un valor de $14,7 \mathrm{~cm} \mathrm{H} 20$ en las pacientes con MC (86 casos), y en las pacientes con MD (24 casos), dicho valor medio fue de $25,3 \mathrm{~cm} \mathrm{H} 20$. A mbas medias se diferenciaron significativamente $(p=0,01)$. Figura 5 . Interpretando el grado medio de obstrucción, según la Chess clasification (PURR; parámetro de resistencia uretral orgánica), las pacientes con MC (83 casos), tuvieron un grado de obstrucción constrictiva de 0,5 frente a 1,1 de las pacientes con MD (22 casos), siendo dichas diferencias muy significativas $(p=0,009)$. (Figura 5).

De igual forma, el grado medio de obstrucción compresiva fue estadísticamente distinto $(p=0,04)$. En el grupo de MC (83 casos) fue de 0,02, mientras que en el grupo de MD, fue de 0,4.(Figura 5 ). $\mathrm{No}$ se demostraron diferencias significativas en el parámetro de resistencia uretral dinámica (DURR), entre ambos grupos.

Los parámetros de resistencia uretral estructural u orgánica, y funcional, no presentaron relación significativa con la presencia y grado de cistocele.

\section{C) Parámetros de Contractilidad vesical.}

\section{.- W80-20.}

Las pacientes con micción compensada 75 casos), tuvieron un valor medio del W 80-20 de 4,08 $y$ en las pacientes con micción descompensada (20 casos), dicho valor arrojó una media de 1,7 . Existieron diferencias muy significativas en la comparación de ambas medias $(p<0,0001)$. (Figura 6$)$.

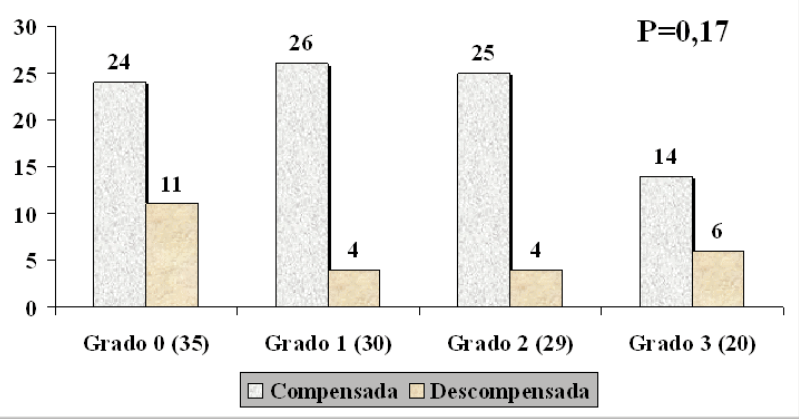

FIG URA 4. Descompensación miccional y grado de cistocele. $N=114$
De igual forma, la interpretación del W 80-20 como positivo o neutro (normal; 105 casos), también arrojó diferencias significativas entre ambos grupos. A sí, las pacientes con micción compensada presentaron W 80-20 positivo o neutro en 62 de 77 casos $(80,5 \%)$ y en las pacientes con micción descompensada lo fue en 4 de las 28 enfermas (14,2\%). Estas diferencias en las proporciones fueron estadísticamente muy significativas $(p<0,000001)$.

La demostración de un W 80-20 negativo (afectación de la contracción isotónica del detrusor) presentó relación significativa $(p=0,02)$ con el grado de cistocele.

\section{-- W max.}

Por el contrario, no se demostraron diferencias significativas con el $\mathrm{W} \max$ (medido en $\mathrm{w} / \mathrm{m}^{2}$ ) entre ambos grupos y grado de cistocele $(p=0,69)$.

\section{-- Duración de la contracción del detrusor.}

El residuo postmiccional medio, del test presión detrusor/ flujo micconal en las pacientes con MC fue de $33,9 \mathrm{ml}$ frente a $101,7 \mathrm{ml}$ en el grupo de MD. Estas medias fueron estadísticamente distintas $(p=0,007)$.

El residuo postmiccional del estudio presión flujo (que nos sirvió para medir la duración de la contracción del detrusor), en los diferentes grados de cistocele siguió la siguiente distribución (Tabla I).

Existieron diferencias significativas en el residuo promedio entre las pacientes con cistocele grado 3 y el resto ( 0 vs. $3 p=0,01 ; 1$ vs. $3 p=0,001 ; 2$ vs. 3 $p=0,02$ ).

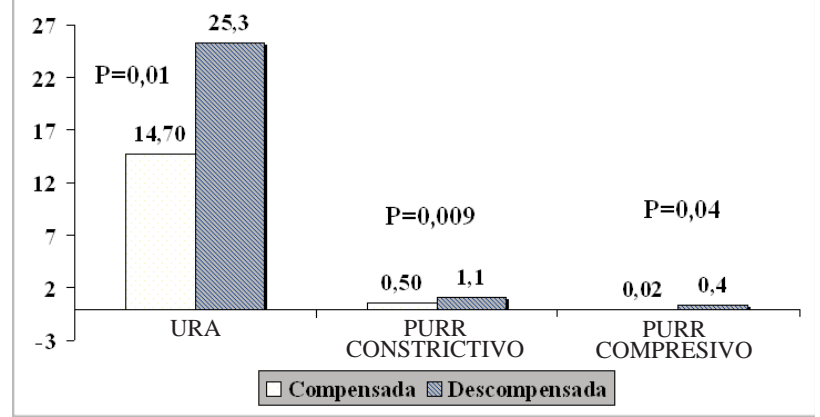

FIG URA 5. Parámetros de resistencia uretra I. 


\section{7.- Datos radiológicos (cistouretrografía).}

Las pacientes del grupo de MC presentaron alteraciones uretrales (distorsión, angulación, dilatación..) en 3 de 30 casos (10\%), mientras que las pacientes con MD, la presentaron en 3 de $6(50 \%)$, siendo estas diferencias significativas $(p=0,01)$, pero no presentó diferencias significativas ni con el grado de cistocele, ni con la obstrucción $(p=0,64)$.

\section{DISCUSIÓN}

En los Síntomas Urinarios del Tracto Urinario Inferior (LUTS), de tipo vaciamiento, únicamente presento diferencias significativas la dificultad miccional, que fue referida mas frecuentemente, en el grupo de micción descompensada, que en el grupo de micción compensada. El resto de los síntomas funcionales, no presentaron diferencias significativas.

En nuestro trabajo, hemos tenido en cuenta fundamentalmente, en la flujometría, el residuo postmiccional, ya que consideramos que los otros datos, como el flujo máximo, nos ayudarían poco al diagnostico de obstrucción del tracto urinario inferior versus afectación contráctil del detrusor. El percentil del flujo máximo si presentó diferencias significativas entre a mbos grupos, aunque el resto de los parámetros de la flujometría, no han demostrado diferencias significativas.

El diagnóstico de obstrucción del tracto urinario inferior en la mujer no es fácil, existiendo diversos parámetros de medida. En nuestro trabajo hemos utilizado el URA y PURR, y recientemente algunos autores han aplicado otros, como el nomograma de Blaivas y Grout (1).

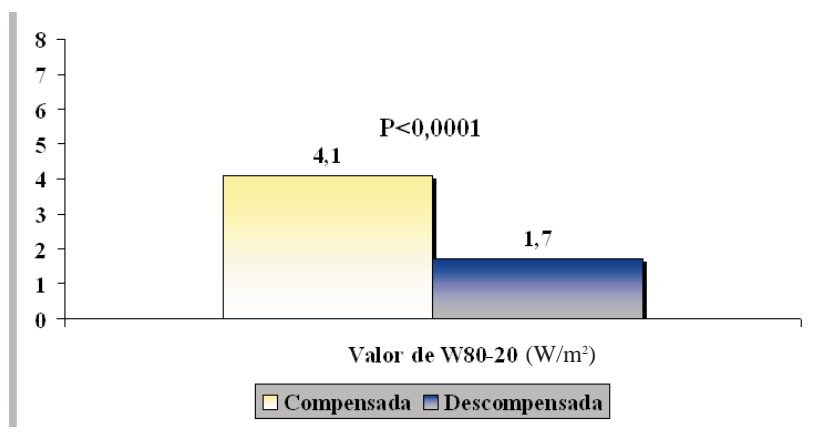

FIG URA 6. Parámetros de contractilidad vesical.
En nuestra serie, la micción descompensada presentó unos valores más altos de resistencia uretral (URA) que el grupo de micción compensada, lo que permitiría atribuir el residuo postmiccional a la existencia de una obstrucción del tracto urinario inferior. La demostración, con carácter significativo, de una hiperactividad vesical más frecuente, en el grupo de micción descompensada, y con relación estadísticamente significativa, con el incremento de la resistencia uretral, apoyaría este diagnostico de obstrucción del tracto urinario inferior, como ya fue descrito en el varón por Salinas et al (2), y Turner-W arwick (3).

La medida del PURR nos permitió tipificar en nuestra serie la obstrucción, más significativamente de carácter estructural constrictivo, similar a la observada en el varón con estenosis uretral (4).

Se descartó la posibilidad de que la obstrucción fuera de tipo funcional, no estructural, (mediante el estudio del DURR y los datos de la electromiografía perineal), esto es, tanto de tipo autonómico como de tipo somático (micción no coordinada) $(5,6)$.

En nuestro estudio, el diagnostico de obstrucción no presentó relación significativa con la presencia y grado de cistocele, lo que coincide en este punto con lo descrito por algunos autores (7), pero no con otros (8), que relacionan el diagnóstico de obstrucción con prolapsos vaginales severos. No obstante, esto podría explicarse porque los criterios de obstrucción, de estos últimos autores, fueron diferentes de los utilizados por nosotros. En ocasiones, los datos de obstrucción en prolapsos severos disminuirían o desaparecerían postreducción del mismo.

0 tros autores (9), llegan así mismo a relacionar la obstrucción con la presencia de prolapsos severos, pero basan el diagnostico de obstrucción en los datos de la flujometría libre, y por otra parte, los prolapsos no se reducían a cistoceles aislados, sino incluyen en su serie otros tipos de prolapsos: prolapso uterino, rectocele y enterocele, que también pueden contribuir a la obstrucción uretral.

A unque los datos de la flujometría libre no son fiables en el diagnóstico de obstrucción, Valentini et al (10), describen un modelo matemático-informático aplicado a la flujometría libre (urodinámica no invasiva), que les permitió identificar en los cistoceles 
una obstrucción de tipo constrictivo, como el encontrado en nuestra serie de micción descompensada, a través de la aplicación del PURR, al estudio invasivo presión/ flujo.

A pesar del diagnóstico de obstrucción en nuestra serie, no siempre fue posible localizar la zona de obstrucción, aunque se utilizara en un alto porcentaje de casos, el estudio radiológico.

En este sentido, en algunos casos, fue demostrable una distorsión uretral, asociado a un marcado descenso vesical durante la micción, que localizaría la obstrucción a dicho nivel, pero en otros casos no fue obtenible ningún dato fiable.

Es posible que en estos últimos casos, se produzca durante la micción una caída del retrotrígono por debajo de la uretra (no perceptible radiológicamente), tal como describe Porena et al (11), conduciendo a la obstrucción del tracto urinario inferior.

0 tro factor a tener en cuenta seria la existencia de diversos tipos de prolapso vaginal (que no fueran el cistocele aislado, condición por otra parte rara): prolapso uterino, prolapso de cúpula, rectocele, enterocele, ... que producirían obstrucción (a nivel del cuello vesical-uretra), siendo por otra parte difícilmente valorables mediante las técnicas radiológicas habituales (cistouretrografías).

A sí, la corrección quirúrgica de los prolapsos, relacionados con obstrucción, se acompaño de una normalización del la flujometría (8), así como de una disminución importante de la hiperactividad vesical secundaria $(12,13)$, similarmente a lo que ocurre en el varón cuando se elimina la obstrucción prostática $(14,15)$.

La otra causa de micción descompensada, aparte de la obstrucción del tracto urinario inferior, es la afectación de la contractilidad vesical. En nuestra serie, se demostró una insuficiencia de la contractilidad isotónica del detrusor (medida mediante el W 80 20), con significación estadística, en el grupo de micción descompensada, que presento relación directa con el grado de cistocele. A sí mismo, el otro parámetro de contractilidad vesical, como es la duración de la contracción del detrusor (medida por el residuo postmiccional del test de presión/ flujo), también se comprobó afectada significativamente, en el grupo de micción descompensada. Esta afectación presento una relación significativa con el grado de cistocele, que fue mucho mas intensa en los cistoceles mas severos (grado III), que en los menos severos, y en ausencia de cistocele (cistocele grado 0). Sin embargo, la contractilidad isométrica del detrusor (W max), no presento ninguna diferencia significativa entre los dos grupos, ni relación con la presencia y grado de cistocele, que fue ya descrito en anterioridad (16). Esto significa que la vejiga tiene una contracción de suficiente intensidad pero de corta duración. Esta afectación de la contractilidad vesical (contracción isotónica y duración de la contracción del detrusor) podría tener carácter pronóstico en la corrección quirúrgica del cistocele.

Esta afectación significativa de la contractilidad vesical (contracción isotónica y duración de la contracción del detrusor), en relación con los cistoceles mas severos, no fue demostrada por Romanzi et al8, que podría ser explicado porque los criterios aplicados al estudio de la contractilidad vesical por este autor fueron diferentes.

Finalmente, la utilización de prensa abdominal, fue demostrada en ambos grupos, no comprobándose diferencias significativas, así como su relación a la presencia y grado de cistocele. Esto es, la aplicación de la prensa abdominal en la micción, no es infrecuente en la mujer, como ya describió Tanagho et al (17), y su demostración no presentaría un carácter de factor de riesgo para la producción de cistocele.

Por el contrario, la utilización de prensa abdominal en la micción, si podría tener significación patológica en el varón, como describió A dot et al (18) en la serie de divertículos vesicales, al comprobar la asociación estadística entre afectación de la contractilidad vesical y micción con prensa abdominal.

\section{BIBUOGRAFIA y LECTURAS RECOMENDADAS (*lectura de interés y **lectura fundamental)}

**1. BLAIVAS, J.G.; GROUTZ, A. : "Bladder outlet obstruction. Nomogram for women with lower urinary tract symptomatology “. Neurourol. Urodyn. 19:553. 2000

2. SALINAS, J.; PRIETO, L.; MARTÍN, C. Y COLS.: “ Utilidad de la aceleración del flujo miccional en el diagnóstico de la inestabilidad vesical". Arch. Esp. Urol. 46:891, 1993

3. TURNER-WARWICK, R.; WHITESIDE, C.G.; WORTH, P.L. y cols.: "A urodynamic view of the cli- 
nical problems associated with bladder neck dysfunction ant its treatment by endoscopic incision and transtrigonal posterior prostatectomy”. Br. J. Urol.45:44. 1973

4. ADOT, J.M.; SALINAS, J. VIRSEDA, M, : "Resultados clínicos, urodinámicos, y radiológicos en el estudio del prostatismo mediante la medida de la PURR (relación de resistencia uretral pasiva)". Arch. Esp.Urol. 48:162. 1995

5. SALINAS, J.; ARISTIZABAL, J.M.; VÍRSEDA, M. Y cols.: "Utilidad del DURR (factor de resistencia uretral dinámica) en el estudio del prostatismo". Arch. Esp. Urol. 49, 4: 405. 1996.

6. SALINAS, J.; VÍRSEDA, M.; ESTEBAN, M., y cols. "Mediur 21.: La implementación de un programa informático/gráfico en Urodinámica." Urod. A. 16:68. 2003

7. ILLIEV, N.V.; LAZAREVSKI, B.; CONEJERO, J. y cols. : " Influencia del cistocele en la alteración urodinámica del vaciado vesical “. Urod. A, 4: 194. 1996

*8. ROMANZI, L.J.; CHAIKIN, D.C.; BLAIVAS ,J.G.: “ The effect of genital prolapse on voiding". J. Urol.161:581.1999

*9. VECC IOLI. SCALDAZZA,C. "Relation between volume of residual urine and urodynamic findings in women with cystourethrocele". Urol. Int. 68:152. 2002

10. VALENTINI, F.; BESSON, G.; NELSON, P. y cols. : "A mathematical micturition model to restore simple flow recording in a healthy and symptomatic individuals and enhance uroflow interpretation". Neurourol. Urodyn 19:153. 2000.

*11. PORENA, M.; BISCOTTO, S.; CONSTANTINI, E. y cols. : "Perugia urodinamic method of analysis (PUMA): a new advanced method of urodynamic analysis applied clinically and compared with other advanced methods". Neurourology and Urodynamics. 22:206. 2003

12. N GUYEN, J.K.; BHATIA, N.M.: " Resolution of motor urge incontinente after surgical repair of pelvic organ prolapse" . J. Urol. 166:2263. 2001

13. ESPUÑA ,M.; PUIG, M.; PEREZ, A.: "Inestabilidad del detrusor asociada al prolapso genital severo: resolución tras el tratamiento quirúrgico". Urod. A. 4: 103. 2001

14. TURNER-WARWICK R. Bladder outflow obstruction in the male. In : MUNDY, A.R.; STHEPHENSON, T.P.; WEIN, A.J. editors. Urodynamics: principles, practice and applications. New York-ChurchillLivingstone. Pp183-204.1994.

15. ABRAMS, P.H..: "Detrusor instability and bladder outlet obstruction". Neurourol Urodyn. 4:317. 1985.

16. ADOT, J.; SALINAS, J.; DAMBROS, M.:" Factores de descompensación miccional y cistocele". Comunicación Congreso Asociación Española de Urología. Oviedo. 2004.

17. TANAGHO, E.A.; STOLLER, M.L. : "Urodynamics: uroflowmetry and female voiding patterns". In ostergard DR, Bent AE (eds). Urogynecology and Urodynamics. Baltimore, Williams and Wilkins, $p$ 164-170. 1991.

18. ADOT, J.; SALINAS, J.; DAMBROS, M.; y cols.:" La urodinámica del divertículo vesical en el varón adulto”. En Arch Esp Urol. (en prensa). 2005. 